\title{
Effect of revascularization on coronary chronic total occlusion in patients undergoing coronary artery bypass grafting
}

\author{
Jiyun Lee ${ }^{1 \#}$, Yun Seok Kim ${ }^{2 \#}$, Hwan Wook Kim ${ }^{3}$, Joon Kyu Kang ${ }^{4}$, Hyun Song ${ }^{3}$, Ju Yong Lim ${ }^{3}$ \\ ${ }^{1}$ Department of Thoracic and Cardiovascular Surgery, Incheon St. Mary's Hospital, College of Medicine, The Catholic University of Korea, \\ Incheon, Republic of Korea; ${ }^{2}$ Department of Thoracic and Cardiovascular Surgery, Keimyung University Dongsan Hospital, Daegu, Republic of \\ Korea; ${ }^{3}$ Department of Thoracic and Cardiovascular Surgery, Seoul St. Mary's Hospital, College of Medicine, The Catholic University of Korea, \\ Seoul, Republic of Korea; ${ }^{4}$ Department of Thoracic and Cardiovascular Surgery, Eunpyeong St. Mary's Hospital, College of Medicine, The Catholic \\ University of Korea, Seoul, Republic of Korea \\ Contributions: (I) Conception and design: All authors; (II) Administrative support: JY Lim; (III) Provision of study materials or patients: HW Kim, \\ JK Kang, H Song, JY Lim; (IV) Collection and assembly of data: J Lee, JY Lim; (V) Data analysis and interpretation: J Lee, YS Kim, JY Lim; (VI) \\ Manuscript writing: All authors; (VII) Final approval of manuscript: All authors. \\ "These authors contributed equally to this work as the first authors. \\ Correspondence to: Ju Yong Lim, MD, PhD. Department of Thoracic and Cardiovascular Surgery, Seoul St. Mary's Hospital, College of Medicine, \\ The Catholic University of Korea, 222 Banpo-daero, Seocho-gu, Seoul 06591, Republic of Korea. Email: millalim92@gmail.com.
}

Background: Bypass grafting for chronic total occlusions (CTOs) remains surgically challenging and controversial. Therefore, we evaluated the incidence and clinical outcomes of revascularization on CTOs undergoing coronary artery bypass grafting (CABG).

Methods: Among 828 patients who underwent isolated CABG from January 2010 to December 2018, 245 patients (29.6\%) diagnosed with at least one CTO were included and retrospectively reviewed. Primary endpoints were 30-day and overall mortality. Secondary endpoint was the composite outcome of major adverse cardiac and cerebrovascular events (MACCE).

Results: With a mean follow-up of $56.6 \pm 6.5$ months in 245 patients with CTOs, 51 patients $(20.8 \%)$ received incomplete revascularization (ICR) for CTO lesions. Risk factor analysis showed that ICR was associated with increased 30-day [odds ratio 8.62; 95\% confidence interval (CI): 1.64-50; $\mathrm{P}=0.011$ ] and overall mortality (hazard ratio (HR) 2.13 ; $95 \%$ CI: $1.07-4.21 ; \mathrm{P}=0.03$ ). ICR also increased the risk of MACCE (HR 1.98; 95\% CI: 1.12-3.54; P=0.01). Freedom from overall mortality was 92.8\%, 90.4\%, and $86.8 \%$ in the complete revascularization group, and $86.3 \%, 80.0 \%$, and $72.7 \%$ in the ICR group, at 1,3 , and 5 years, respectively $(\mathrm{P}=0.004)$.

Conclusions: In patients with CTOs undergoing CABG, the rate of ICR was $20.8 \%$, and it significantly increased the risk of mortality and MACCE. Further studies in a large cohort are needed.

Keywords: Coronary artery bypass grafting (CABG); chronic total occlusion of coronary artery (CTO of coronary artery); complete revascularization

Submitted Apr 17, 2021. Accepted for publication Sep 03, 2021.

doi: $10.21037 /$ jtd-21-681

View this article at: https://dx.doi.org/10.21037/jtd-21-681

$\wedge$ ORCID: 0000-0002-2817-557X.

(C) Journal of Thoracic Disease. All rights reserved. 


\section{Introduction}

Chronic total occlusions (CTOs) of coronary arteries are an exacerbation of stable coronary artery disease with advanced calcification (1). CTOs are present in up to one third of patients undergoing coronary angiography (2). Even though various degree of collateral vessels develop and contribute to the perfusion of CTO territories, revascularization on CTOs was attempted to relieve angina or heart failure with its chronic progressing nature (3).

One of the options for revascularization is percutaneous coronary intervention (PCI), one advantage of which is that it is done under local anesthetics, postprocedural morbidity is minimal, and patients endure a short hospital stay (4). Successful PCI is associated with a significant decrease in both short- and long-term mortality (5). However, PCI of CTO remains a challenge and delivers worse results because of the lower initial success rate with angioplasty and a higher frequency of restenosis or reocclusion. The other alternative is revascularization by coronary artery bypass grafting (CABG). Since there is generally no difference in the techniques of revascularization whether the vessel is highly stenosis or completely occluded (6), the presence of CTO may not affect the complexity of CABG or postoperative outcomes, including the incidence of major adverse cardiac and cerebrovascular events (MACCE) $(7,8)$.

Even though several studies of revascularization on demonstrated favorable outcomes of CABG over PCI $(4,8)$, bypass grafting remains surgically challenging. Especially, the lower rate of successful surgical revascularization has been associated with features including the presence of occlusion at a side branch, heavy calcification, and a length of CTO of more than $50 \mathrm{~mm}(2,9)$. In addition, there is lack of evidence on the survival benefit of complete revascularization for CTOs in patient undergoing CABG. Therefore, in the following study, we sought to evaluate the incidence of revascularization for CTOs and its clinical outcomes in patients undergoing CABG.

We present the following article in accordance with the STROBE reporting checklist (available at https://dx.doi. org/10.21037/jtd-21-681).

\section{Methods}

\section{Study design and subjects}

Among 828 patients who underwent isolated CABG at a single institution from January 2010 to June 2018, 245 patients $(29.6 \%)$ diagnosed with at least one CTO were retrospectively reviewed from the electronic medical records. We divided the cohort into two groups, according to the degree of revascularization: the complete revascularization (CR) group, in which the patients received revascularization for all CTO lesions; and the incomplete revascularization (ICR) group, in which at least one CTO lesion remained unbypassed. Surgical decisions for revascularization were made by the primary surgeon based on the quality of the conduit, the diameter of the native coronary artery, the degree of calcification, and the viability of the myocardium.

Primary endpoints were 30-day and overall mortality. The secondary endpoint was the composite outcome of MACCE.

The study was conducted in accordance with the Declaration of Helsinki (as revised in 2013). This study was approved by the Institutional Review Board of Seoul St. Mary's Hospital, Seoul, Republic of Korea (IRB number: KC20RASI0157) and individual consent for this retrospective analysis was waived. There was no funding source used for the study. The authors declare that they have no competing interests. The datasets used and analyzed during the current study are available from the corresponding author on reasonable request.

\section{Definitions and variables}

CTO was defined as $100 \%$ luminal diameter stenosis without a discernable lumen, the absence of antegrade flow, known or assumed to be $\geq 3$ months' duration, based on the prior angiography, and the presence of bridging collaterals or concordant acute coronary syndrome. The angiographic data were reviewed and interpreted by cardiologists in the coronary angiographic laboratory. All CTOs in the left anterior descending coronary artery (LAD) and the major diagonal branches were grouped as CTOs in the LAD distribution. Similarly, CTOs in the left circumflex (LCX) and the major obtuse marginal branches were classified as the LCX distribution CTOs. CTOs in the right coronary artery (RCA) and its major branches were classified as CTOs in the RCA territory. Good collateral was defined as good coronary collateralization based on the Rentrop classification, grade of 2 or 3. According to the Rentrop classification, the degree of recipient artery contrast filling is used to classify collateral vessels: grade 0 , no collateral; grade 1, recipient side branch filling only; grade 2, partial filling of main recipient vessel; and grade 3, complete filling of main epicardial recipient vessel. Thirty-day and overall mortality included all causes of death. MACCE was defined as all-cause mortality, stroke or transient ischemic 


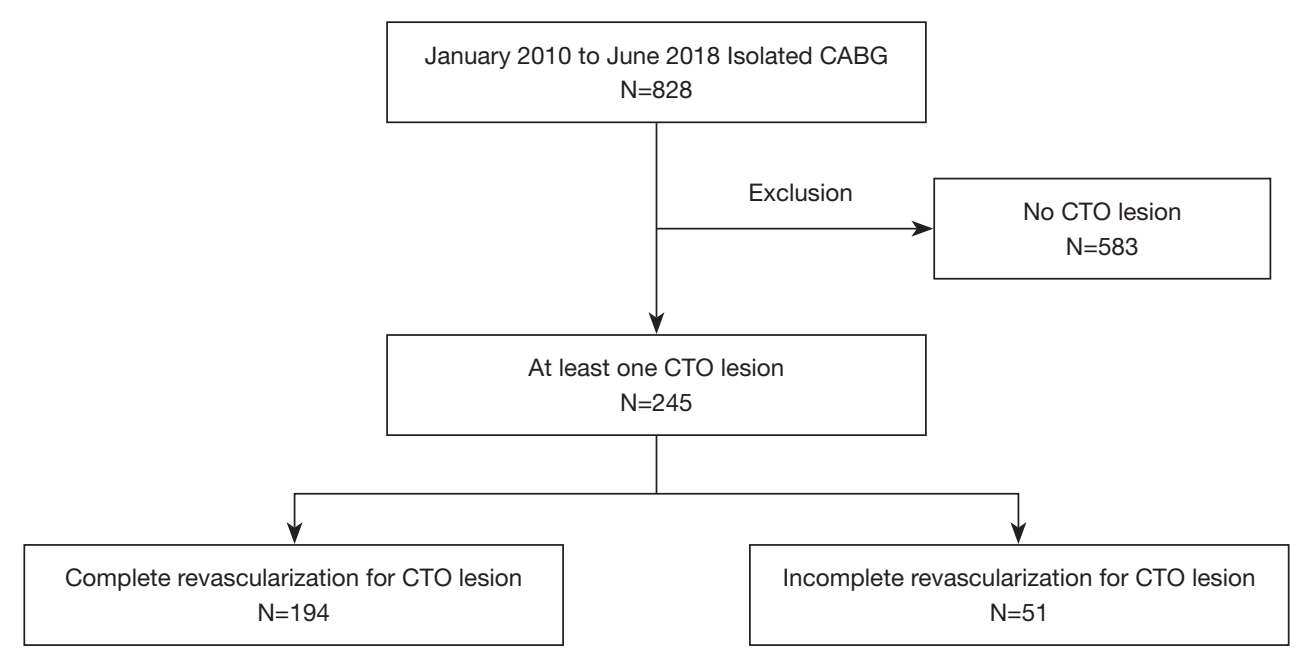

Figure 1 Flow diagram of the study. CABG, coronary artery bypass grafting; CTO, chronic total occlusion.

attack, and acute coronary syndrome requiring repeated revascularization.

Follow-up information about outcomes was obtained from out-patient visits, telephone interviews, and medical records of the national health insurance.

\section{Surgical revascularization of CTOs}

In our institution, we primarily perform conventional CABG with standard cardiopulmonary bypass and cardiac arrest, but off-pump CABG was considered in patients with significant atherosclerotic aortic disease. Decisions for whether to bypass a CTO lesion or not were mainly based on the quality of the conduit, the anatomical complexity such as the diameter of the native coronary artery, the degree of calcification. Severely calcified CTO lesion requiring thromboendarterectomy was not revascularized. Left internal mammary artery (LIMA) was the first choice of conduit to revascularize CTOs in LAD territory. For non-LAD territory, we selected the radial artery over the saphenous vein graft, especially for LCX territory to revascularize CTOs.

The intraoperative mean graft flow and pulsatility index (PI) were measured with a transit time flow measurement (TTFM) device (MedStim, Oslo, Norway) for all anastomosis at the time of CPB weaning or haemodynamic stabilization with a systolic blood pressure of 100-120 mmHg. According to the European Association for Cardiothoracic Surgery and European Society of Cardiology (EACTS/ESC) guideline, we followed the TTFM cut-off threshold values; mean flow $\geq 20 \mathrm{~mL} / \mathrm{min}$, $\mathrm{PI} \leq 5$. If TTFM value shows suboptimal or outside the threshold value, then we consider to revise the anastomosis.

\section{Statistical analysis}

Data are expressed as mean \pm standard deviation for continuous variables and as number and percentages for categorical variables. Continuous parameters were compared with Student's $t$-test, and discrete variables were compared using the $\chi^{2}$ test or the Fisher's exact test, as appropriate. Regarding the risk factor analysis for 30-day mortality, only univariate analysis using logistic regression was performed due to the small event number $(\mathrm{n}=9)$. We did univariate and multivariate analyses for the entire patient cohort using the Cox proportional hazards model for overall mortality and MACCE. We included the most clinically relevant variables in the multivariate analysis if their univariate significance was $\mathrm{P} \leq 0.1$, and used the backward elimination method for variable selections. Cumulative event rates and survival curves were generated using the Kaplan-Meier method with the logrank test. The critical value of $\mathrm{P} \leq 0.05$ was used for statistical significance. We did all analyses with IBM SPSS Statistics for Windows, Version 24.0 (IBM Corp., Armonk, NY, USA).

\section{Results}

\section{Baseline characteristics}

With a mean follow-up duration of $56.6 \pm 6.5$ months in 245 patients with CTOs who underwent isolated CABG, 51 patients $(20.8 \%)$ received ICR for CTO lesions (Figure 1). Baseline characteristics and comparison of the 
Table 1 Baseline characteristics

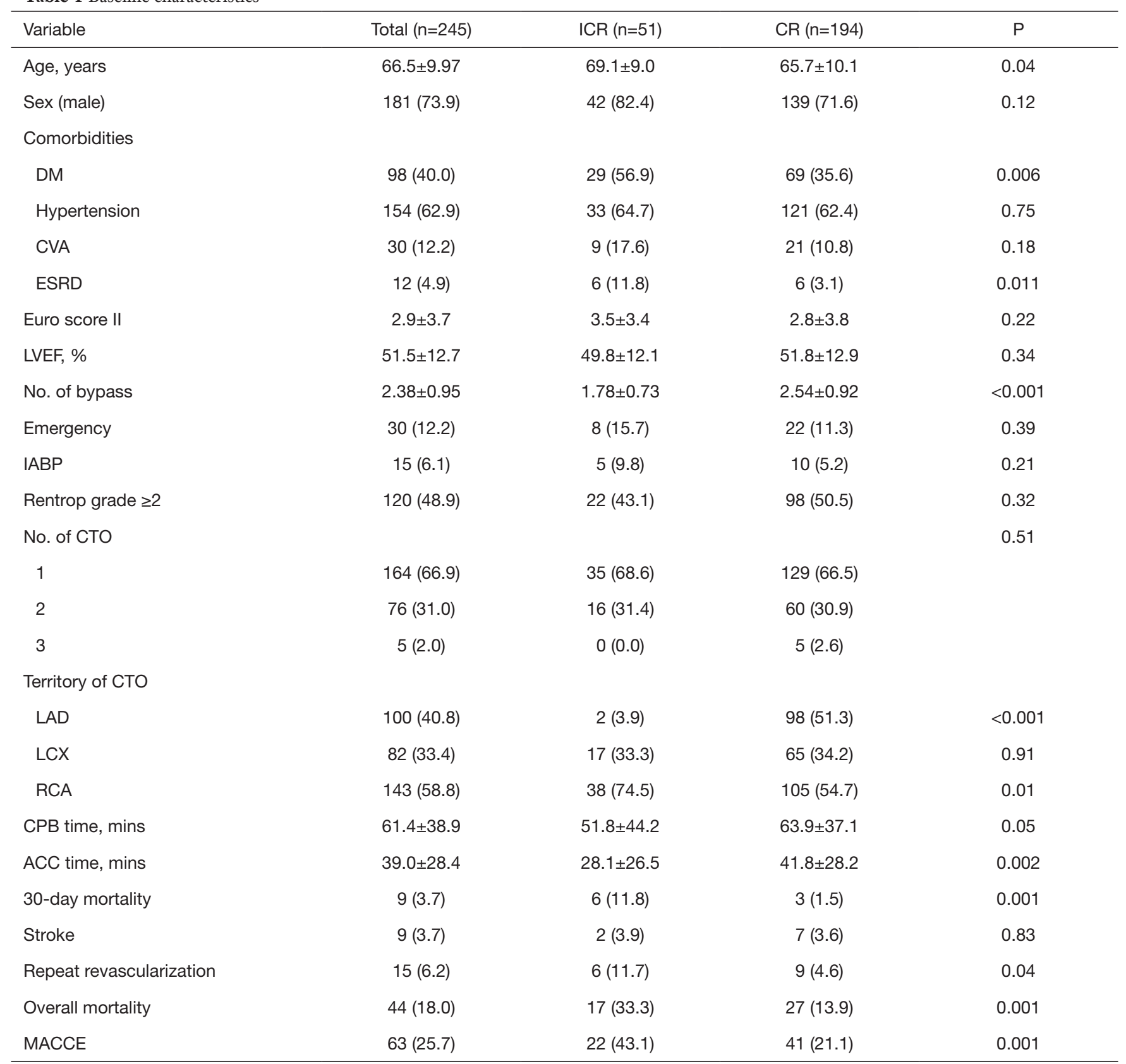

Data are represented by mean \pm SD or $n(\%)$. ACC, aortic cross clamp; CPB, cardiopulmonary bypass; CR, complete revascularization; CTO, chronic total occlusion; CVA, cerebrovascular accident; DM, diabetes mellitus; ESRD, end-stage renal disease; IABP, intra-aortic balloon pump; ICR, incomplete revascularization; LAD, left anterior descending artery; LCX, left circumflex artery; LVEF, left ventricular ejection fraction; MACCE, major adverse cardiac and cerebrovascular event; No., number; RCA, right coronary artery.

two groups are summarized in Table 1. In the ICR group, patients were older $(\mathrm{P}=0.04)$, had more comorbidities, like diabetes $(\mathrm{P}=0.006)$ and end-stage renal disease $(\mathrm{P}=0.011)$.

Regarding CTO territories, the RCA territory were involved predominantly in both groups (ICR vs. CR, 74.5\% vs. $54.7 \%$, respectively). The rate of CR was higher in the LAD territory $(3.9 \%$ vs. $51.3 \%, \mathrm{P}<0.001)$, but lower in the RCA territory $(74.5 \%$ vs. $54.7 \%, \mathrm{P}=0.01)$. The ICR 
Table 2 Univariate analysis for 30-day mortality

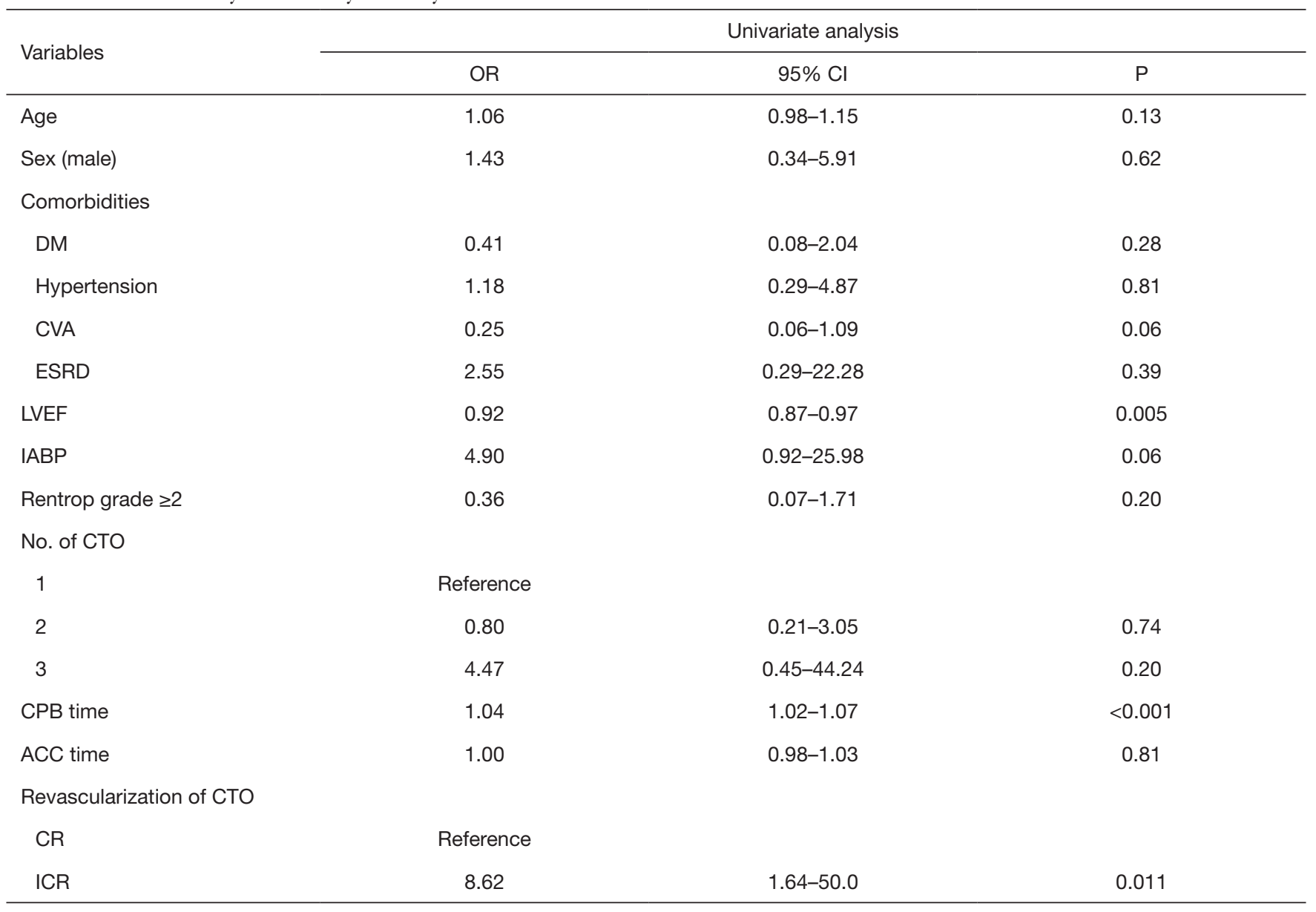

ACC, aortic cross clamp; Cl, confidence interval; CPB, cardiopulmonary bypass; CR, complete revascularization; CTO, chronic total occlusion; CVA, cerebrovascular accident; DM, diabetes mellitus; ESRD, end-stage renal disease; IABP, intra-aortic balloon pump; ICR, incomplete revascularization; LVEF, left ventricular ejection fraction; MACCE, major adverse cardiac and cerebrovascular event; No., number; OR, odds ratio.

group showed higher mortality at 30 days $(11.8 \%$ vs. $1.5 \%$, $\mathrm{P}=0.001)$ and overall (33.3\% vs. $13.9 \%, \mathrm{P}=0.001)$, and higher incidence of MACCE ( $43.1 \%$ vs. $21.1 \%, \mathrm{P}=0.001$ ).

\section{Risk factor analyses for outcomes}

In the analysis of risk factors for 30-day mortality, longer cardiopulmonary bypass time [odds ratio (OR) 1.04; $95 \%$ confidence interval (CI): 1.02-1.07; $\mathrm{P}<0.001]$ and low left ventricular ejection fraction (LVEF) (OR 0.92; 95\% CI: $0.87-0.97 ; \mathrm{P}=0.005$ ) were identified as significant risk factors. Also, ICR for CTO lesions was associated with increased risk for 30-day mortality (OR 8.62; $95 \%$ CI: 1.64-50; $\mathrm{P}=0.011$ ) (Table 2).

In the analysis of risk factors for overall mortality, the multivariate analysis showed that the ICR was significantly associated with increased overall mortality [hazard ratio (HR) 2.13; 95\% CI: $1.07-4.21 ; \mathrm{P}=0.03$ ] as well as age (HR 1.03; 95\% CI: $1.00-1.07 ; \mathrm{P}=0.03$ ) (Table 3).

In the analysis of risk factors for MACCE, low LVEF (HR 0.96; 95\% CI: 0.94-0.99; $\mathrm{P}=0.01$ ) and ICR for CTOs (HR 1.98; 95\% CI: 1.12-3.54; $\mathrm{P}=0.01$ ) were associated with increased risk for MACCE (Table 4).

\section{Freedom from overall mortality and MACCE between groups}

Freedom from overall mortality and MACCE was significantly higher in the CR group. Freedom from overall mortality was $92.8 \%, 90.4 \%$, and $86.8 \%$ in the CR group, 
Table 3 Univariate and multivariate analyses for overall mortality

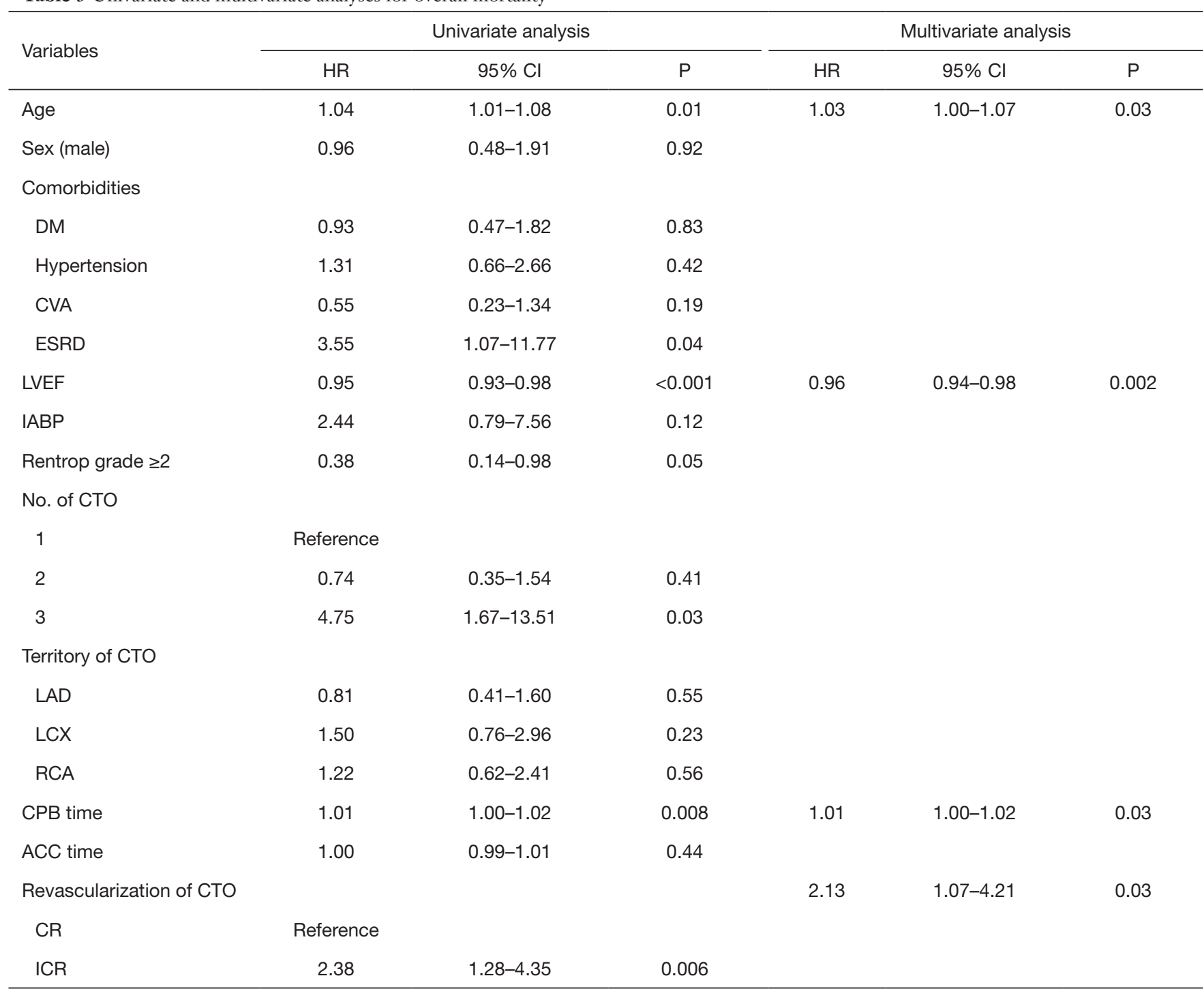

ACC, aortic cross clamp; Cl, confidence interval; CPB, cardiopulmonary bypass; CR, complete revascularization; CTO, chronic total occlusion; CVA, cerebrovascular accident; DM, diabetes mellitus; ESRD, end-stage renal disease; HR, hazard ratio; IABP, intra-aortic balloon pump; ICR, incomplete revascularization; LAD, left anterior descending artery; LCX, left circumflex artery; LVEF, left ventricular ejection fraction; MACCE, major adverse cardiac and cerebrovascular event; No., number; RCA, right coronary artery.

and $86.3 \%, 80.0 \%$, and $72.7 \%$ in the ICR group, at 1,3 , and 5 years, respectively $(\mathrm{P}=0.005)$ (Figure 2). Freedom from MACCE was $91.3 \%, 83.4 \%$, and $77.6 \%$ in the CR group, and $79.8 \%, 72.7 \%$, and $53.6 \%$ in the ICR group at 1 , 3 , and 5 years, respectively $(\mathrm{P}=0.003)$ (Figure 3).

\section{Discussion}

The main findings of this study can be summarized as follows. First, CTO is found in about $29 \%$ of patients undergoing CABG. Among them, $80 \%$ of patients received complete bypass grafting for all CTOs. Second, ICR for CTO lesions was associated with increased 30-day and overall mortality. Also, ICR was related to increased MACCE after bypass grafting for CTOs. The rates of CR for CTOs were $86 \%$ in Fefer et al., nearly $85 \%$ in Banerjee et al., and $80 \%$ in our study, which was similar to those previous studies $(9,10)$.

In our study, ICR for CTOs was one of the independent risk factors for 30-day and overall mortality. Unlike our 
Table 4 Univariate and multivariate analyses for MACCE

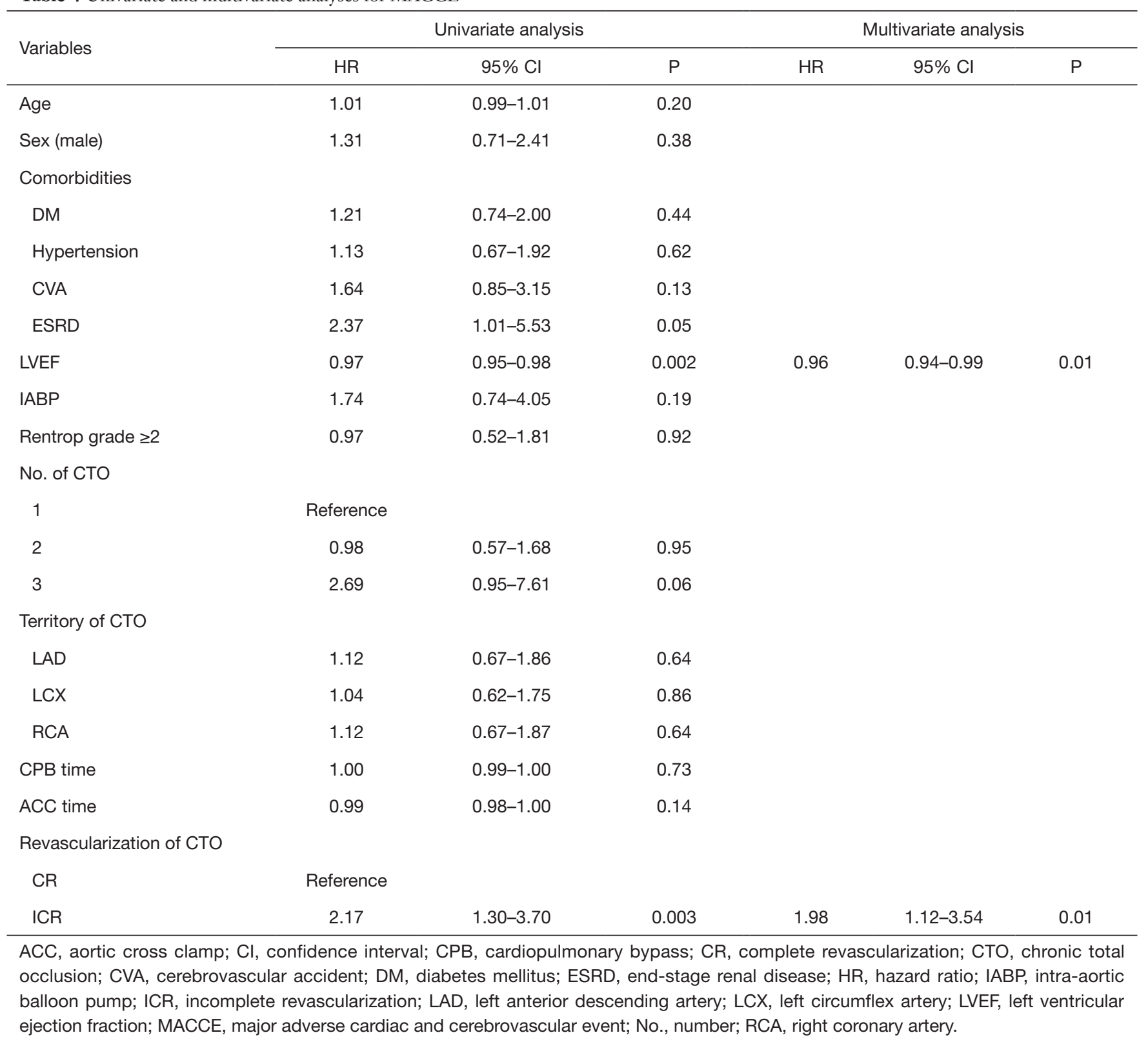

study, Fefer et al. showed that ICR for CTOs was not associated with increased long-term mortality (10). The following theories were supported by the conclusion of their study; the territory supplied by CTO can be non-viable, all non-revascularized territories were in the RCA and LCX territories, and the collateral flow often exists to CTO territory. However, their study lacked assessment of viability and had limited long-term follow-up data. In contrast, Kleisli et al. demonstrated that patients who underwent CABG with ICR had worse outcomes than did patients with
CR (11). Data from SYNTAX also showed that patients with ICR had more adverse events than did patients with CR (12). Therefore, ICR for CTOs was associated with short- and long-term outcomes, as a negative factor (4). As a result, the overall survival in the ICR group for CTOs was significantly lower than that in the CR group.

Farooq et al. concluded that ICR for CTO lesions was associated with significantly higher frequencies of all-cause revascularization and MACCE than was CR (13). These findings are similar to the results shown in our study. Target 


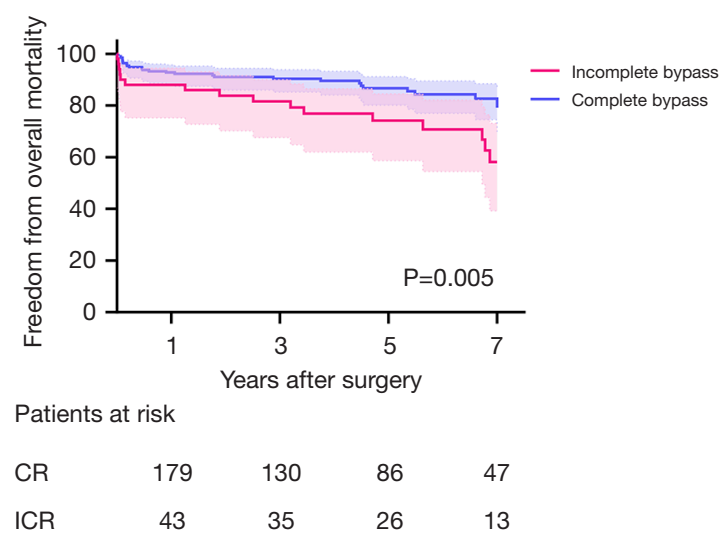

Figure 2 Freedom from overall mortality between the two groups: CR (blue line) and ICR (red line). CR, complete revascularization; ICR, incomplete revascularization.

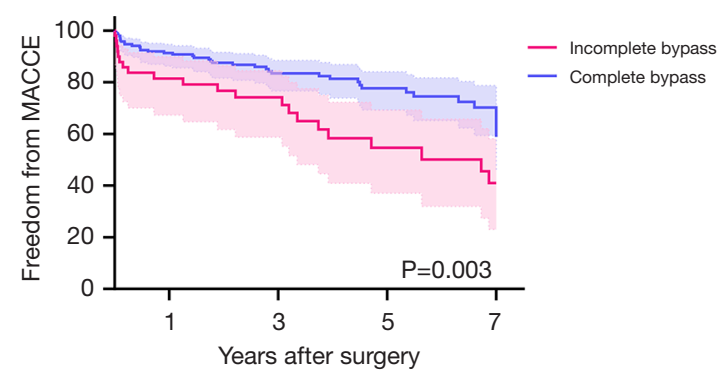

Patients at risk

$\begin{array}{lllll}\text { CR } & 156 & 94 & 56 & 31 \\ \text { ICR } & 36 & 25 & 15 & 8\end{array}$

Figure 3 Freedom from MACCE between the two groups: CR (blue line) and ICR (red line). MACCE, major adverse cardiac and cerebrovascular event; CR, complete revascularization; ICR, incomplete revascularization.

vessel revascularization in the ICR group was significantly higher, as shown in Figure 4. This event seemed to occur mostly within about 4 years after CABG.

In patients with CTO lesions, the possibility of CR was affected by many other factors, such as the revascularization for CTOs driven by the patient's general condition and the angiographic findings. In this study, patients in the ICR group were older and had more comorbidities, such as diabetes and renal replacement, than did the CR group. According to other literature, patients with ICR were also significantly older and had higher prevalence of comorbidities, including diabetes, hypertension, and heart failure $(9,10)$. Also, an anatomical coronary complexity with

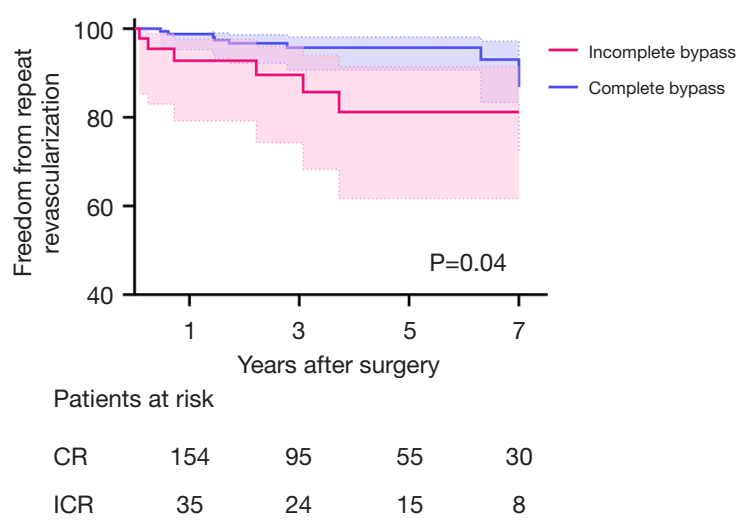

Figure 4 Freedom from repeat revascularization on target vessel after CABG between the two groups: CR (blue line) and ICR (red line). CR, complete revascularization; ICR, incomplete revascularization; CABG, coronary artery bypass grafting.

multiple long and diseased or small segments could have influenced the result of revascularization. Revascularization in patient with this sort of anatomical complexity and poor general condition may be a challenge, and longer surgical procedures are associated with higher postoperative morbidities, longer periods of hospitalization, and slower return to normal activities (4).

There are several limitations in this study. First, it is a retrospective study in a singles institution with a small cohort. Given its observational nature and small sample size, potential confounders might have remained. However, incidence of CTOs was uncommon, so the number of patients with CTOs in our study was similar to that in previous studies for CTOs in CABG. Next, we did not evaluate the viability of the myocardium on CTO lesions routinely unless the myocardial thickness was maintained. Because assessment of viability in all patients undergoing CABG is not cost effective and has limited and uncertain accuracy, with uncertain false-positive and false-negative rates, uncertain prevalence of hibernating myocardium, and thus uncertain predictive values (12). Finally, we collected just hard endpoint follow-up data, like readmission due to heart failure and cardiac function on transthoracic echocardiography, so there is limited information on the relief of angina, history of repeated PCI, and MACCE during the follow-up period.

\section{Conclusions}

In patients with CTO lesions undergoing CABG, the 
rate of ICR was $20.8 \%$, and incomplete revascularization for CTOs was significantly associated with increased risk of MACCE, and 30-day and overall mortality. Further studies with a larger cohort may be necessary to validate this issue.

\section{Acknowledgments}

Funding: None.

\section{Footnote}

Reporting Checklist: The authors have completed the STROBE reporting checklist. Available at https://dx.doi. org/10.21037/jtd-21-681

Data Sharing Statement: Available at https://dx.doi. org/10.21037/jtd-21-681

Peer Review File: Available at https://dx.doi.org/10.21037/ jtd-21-681

Conflicts of Interest: All authors have completed the ICMJE uniform disclosure form (available at https://dx.doi. org/10.21037/jtd-21-681). The authors have no conflicts of interest to declare.

Ethical Statement: The authors are accountable for all aspects of the work in ensuring that questions related to the accuracy or integrity of any part of the work are appropriately investigated and resolved. The study was conducted in accordance with the Declaration of Helsinki (as revised in 2013). This study was approved by the Institutional Review Board of Seoul St. Mary's Hospital, Seoul, Republic of Korea (IRB number: KC20RASI0157) and individual consent for this retrospective analysis was waived.

Open Access Statement: This is an Open Access article distributed in accordance with the Creative Commons Attribution-NonCommercial-NoDerivs 4.0 International License (CC BY-NC-ND 4.0), which permits the noncommercial replication and distribution of the article with the strict proviso that no changes or edits are made and the original work is properly cited (including links to both the formal publication through the relevant DOI and the license). See: https://creativecommons.org/ licenses/by-nc-nd/4.0/.

\section{References}

1. Zakkar M, George SJ, Ascione R. Should Chronic Total Occlusion Be Treated With Coronary Artery Bypass Grafting? Chronic Total Occlusion Should Be Treated With Coronary Artery Bypass Grafting. Circulation 2016;133:1807-16.

2. Braden GA. Chronic total coronary occlusions. Cardiol Clin 2006;24:247-54, vi-vii.

3. Grantham JA, Marso SP, Spertus J, et al. Chronic total occlusion angioplasty in the United States. JACC Cardiovasc Interv 2009;2:479-86.

4. Ong AT, Serruys PW. Complete revascularization: coronary artery bypass graft surgery versus percutaneous coronary intervention. Circulation 2006;114:249-55.

5. Pancholy SB, Boruah P, Ahmed I, et al. Meta-analysis of effect on mortality of percutaneous recanalization of coronary chronic total occlusions using a stent-based strategy. Am J Cardiol 2013;111:521-5.

6. Holzhey DM, Jacobs S, Walther T, et al. Is chronic total coronary occlusion a risk factor for long-term outcome after minimally invasive bypass grafting of the left anterior descending artery? Ann Thorac Surg 2010;89:1496-501.

7. Taggart DP. Incomplete revascularization: appropriate and inappropriate. Eur J Cardiothorac Surg 2012;41:542-3.

8. Kim BS, Yang JH, Jang WJ, et al. Clinical outcomes of multiple chronic total occlusions in coronary arteries according to three therapeutic strategies: Bypass surgery, percutaneous intervention and medication. Int J Cardiol 2015;197:2-7.

9. Banerjee S, Master RG, Peltz M, et al. Influence of chronic total occlusions on coronary artery bypass graft surgical outcomes. J Card Surg 2012;27:662-7.

10. Fefer P, Gannot $S$, Kochkina K, et al. Impact of coronary chronic total occlusions on long-term mortality in patients undergoing coronary artery bypass grafting. Interact Cardiovasc Thorac Surg 2014;18:713-6.

11. Kleisli T, Cheng W, Jacobs MJ, et al. In the current era, complete revascularization improves survival after coronary artery bypass surgery. J Thorac Cardiovasc Surg 2005;129:1283-91.

12. Weintraub WS, Garratt KN. Should Chronic Total Occlusion Be Treated With Coronary Artery Bypass Grafting? Chronic Total Occlusion Should Not Routinely Be Treated With Coronary Artery Bypass Grafting. Circulation 2016;133:1818-25. 
13. Farooq V, Serruys PW, Garcia-Garcia HM, et al. The negative impact of incomplete angiographic revascularization on clinical outcomes and its association with total occlusions: the SYNTAX (Synergy Between

Percutaneous Coronary Intervention with Taxus and

Cardiac Surgery) trial. J Am Coll Cardiol 2013;61:282-94.

Cite this article as: Lee J, Kim YS, Kim HW, Kang JK, Song H, Lim JY. Effect of revascularization on coronary chronic total occlusion in patients undergoing coronary artery bypass grafting. J Thorac Dis 2021;13(11):6343-6352. doi: 10.21037/jtd21-681 